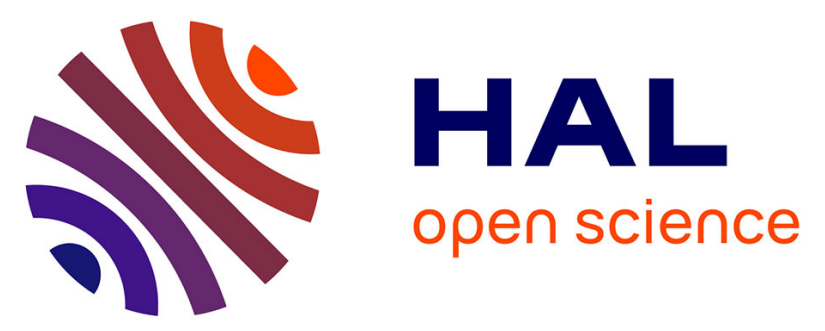

\title{
Spectroscopie d'impédance électrochimique appliquée à l'étude de la corrosion
}

\author{
Claude Gabrielli, Michel Keddam, Hisasi Takenouti
}

\section{To cite this version:}

Claude Gabrielli, Michel Keddam, Hisasi Takenouti. Spectroscopie d'impédance électrochimique appliquée à l'étude de la corrosion. Matériaux \& Techniques, 2007, 95, pp.385 - 395. 10.1051/mattech:2008035 . hal-01731362

\section{HAL Id: hal-01731362 \\ https://hal.sorbonne-universite.fr/hal-01731362}

Submitted on 14 Mar 2018

HAL is a multi-disciplinary open access archive for the deposit and dissemination of scientific research documents, whether they are published or not. The documents may come from teaching and research institutions in France or abroad, or from public or private research centers.
L'archive ouverte pluridisciplinaire HAL, est destinée au dépôt et à la diffusion de documents scientifiques de niveau recherche, publiés ou non, émanant des établissements d'enseignement et de recherche français ou étrangers, des laboratoires publics ou privés. 


\section{Spectroscopie d'impédance électrochimique appliquée à l'étude de la corrosion}

\section{Gabrielli, M. Keddam et H. Takenouti}

UPR 15 du CNRS "Laboratoire Interfaces et Systèmes Électrochimiques (LISE) ", UPMC Univ. Paris 06, Case 133, 4 place Jussieu, 75252 Paris Cedex 05, France

e-mail : claude.gabrielli@upmc.fr; michel.keddam@upmc.fr; hisasi.takenouti@upmc.fr

\section{Mots-clés :}

Impédance faradique; inhibiteur; relaxation; bronze; patrimoine culturel

\section{Key words:}

Faradic impedance; inhibitor; relaxation; bronze; cultural heritage
Résumé - Nous allons présenter différentes applications de la spectroscopie d'impédance électrochimique réalisées depuis 40 ans. Au début de cette longue histoire, l'emploi de signaux alternatifs était destiné à remplacer la méthode stationnaire dite de Stern et Geary, c'est-à-dire la mesure de la résistance de polarisation. Cependant, rapidement nous avons constaté que le spectre d'impédance contenait plusieurs informations sur les phénomènes se déroulant à l'interface métal | électrolyte. Ils se manifestent par une constante de temps propre à chaque processus, donc généralement distinguables par une analyse en fréquence. Après avoir présenté la différence majeure entre la méthode stationnaire et la méthode d'impédance dans l'étude de la corrosion à la fois en termes de compréhension des mécanismes et de prévision de sa vitesse, nous aborderons plusieurs applications de cette dernière méthode : la résistance d'électrolyte, l'effet protecteur d'inhibiteurs organiques et la protection de bronzes archéologiques recouverts de patine.

Abstract - Electrochemical Impedance spectroscopy applied to the corrosion study. Various applications of the Electrochemical Impedance Spectroscopy over the last 40 years are described. At the beginning of this long history, ac signal has been used simply to determine steady state entity, the polarisation resistance to apply Stern-Geary method. However, it was remarked immediately that the impedance spectrum contains many useful information on the processes taking place at the corroding interface. These processes are revealed by a specific time-constant for each phenomenon, therefore they can be distinguished by analysing the impedance spectrum in the frequency domain. After describing the main difference between the steady-state and impedance methods for the corrosion investigation for the understanding of corrosion mechanism as well as the estimation of corrosion rate, we will show several aspects of the latter method: Electrolyte resistance, protective effect of corrosion inhibitor and the protection of bronze archaeological artefacts covered with patina.

a méthode d'impédance électrochimique, souvent appelée spectroscopie d'impédance électrochimique (S.I.E.) est de nos jours très largement utilisée à l'étude de corrosion. Â l'origine, la S.I.E. est fortement orientée vers l'investigation fondamentale, plus particulièrement, à l'étude mécanistique de processus électrochimiques, et cette méthode reste toujours un outil de choix pour cette investigation. En effet, elle est basée sur la réponse linéaire à une perturbation d'une interface électrochimique à étudier, ce qui facilite grandement le développement mathématique des équations décrivant l'évolution temporelle, ou son dual en fréquence : bilan de charge et bilan de matière. La corrosion est un cas particulier de cette application, le courant global est nul et par conséquent on ne peut en extraire aucune information pertinente, d'où le développement de techniques cherchant à tirer partie de la réponse de part et d'autre du potentiel de corrosion.

Initialement, le signal alternatif a été introduit pour remplacer le relevé stationnaire de la courbe courant-potentiel plus long à obtenir et moins reproductible. En 1963, Schwenk et Buhler ont utilisé le signal sinusoïdal de fréquence de 0 à $100 \mathrm{~Hz}$ pour l'évaluation de la corrosion des alliages $\mathrm{Ni}-\mathrm{Cr}$ dans $1 \mathrm{M} \mathrm{H}_{2} \mathrm{SO}_{4}$ [1], et ont décrit leurs résultats par la relation suivante :

$$
j_{\text {corr }}=K^{\prime}+\frac{B}{R_{\mathrm{p}}} \text {. }
$$




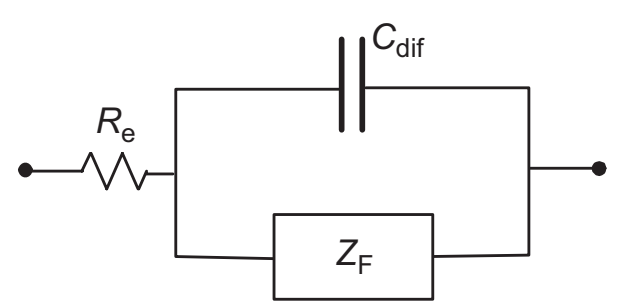

Fig. 1. Circuit équivalant de Randles généralisé à l'impédance faradique $Z_{\mathrm{F}}$. $R_{\mathrm{e}}$ représente la résistance d'électrolyte et $C_{\mathrm{dif}}$ la capacité différentielle de l'interface.

Fig. 1. Generalized Randles equivalent circuit: $R_{\mathrm{e}}$, $C_{\text {dif }}, Z_{\mathrm{F}}$ represents respectively the electrolyte resistance, the interface differential capacitance (double layer capacitance), and the faradic impedance.

Un peu plus tard, 1967, Pražak et Barton [2] ont employé un signal carré de 0,5 à $5 \mathrm{~Hz}$ pour la corrosion d'Ag dans $0,1 \mathrm{M} \mathrm{H}_{2} \mathrm{SO}_{4}$ en présence de différentes concentrations de $\mathrm{Fe}_{2}\left(\mathrm{SO}_{4}\right)_{3}$ et ont introduit une relation différente de l'équation (1).

$$
I_{\text {corr }}=\frac{0,1}{R_{\mathrm{p}}^{\mathrm{k}}} \text {. }
$$

La valeur de l'exposant $k$ varie de 1,2 à 1,9 lorsque la fréquence de perturbation passe de 0,5 à $5 \mathrm{~Hz}$.

Ces auteurs ont donc bien constaté une dépendance de la réponse en courant avec la fréquence de perturbation même si leur idée de départ était bien la mesure de la résistance de polarisation $R_{\mathrm{p}}$. Avec le recul du temps, nous comprenons mieux la raison de ces écarts et leur relation avec l'impédance.

Le comportement dynamique de l'interface peut être représenté par la figure 1 avec le circuit de Randles généralisé [3].

Alors, ils ont mesuré le module d'impédance composé de la résistance d'électrolyte $R_{\mathrm{e}}$ correspondant approximativement à la constante $K^{\prime}$ introduite par Schwenk et Buhler et son couplage avec la capacité de double couche différentielle $C_{\text {dif }}$ et la $R_{\mathrm{p}}$ en parallèle. Ceci justifie la relation (1). Dans le cas de Pražak et Barton, l'équation (1) peut être représentée approximativement par l'équation (2) dans un certain domaine de $R_{\mathrm{p}}$ et $I_{\text {corr }}$.

L'étude de la cinétique électrochimique repose sur l'analyse de $Z_{\mathrm{F}}$ (voir Fig. 1), cependant pour les différents systèmes relevant de la corrosion, les analyses des autres paramètres ne sont pas à négliger. L'application de la méthode d'impédance à la corrosion oblige à adopter la vision la plus large possible des divers éléments qui interviennent dans la réponse en courant (ou en potentiel) de l'interface sous l'effet d'une perturbation de potentiel (ou de courant).

Nous allons d'abord voir le principe sur lequel repose l'étude cinétique de la corrosion par l'impédance électrochimique.

\section{Principe \\ Régime stationnaire}

Wagner et Traud [4] ont découvert empiriquement la relation entre la pente de la courbe courant-potentiel au voisinage de la densité du courant zéro et la vitesse de corrosion, en plus de la notion des cellules locales. Par la suite, cette constatation est confirmée par Bonhoeffer et Jena [5]. C'est un peu plus tard que Stern et Geary ont démontré la relation entre la résistance de polarisation $R_{\mathrm{p}}$ et la densité courant de corrosion $j_{\text {corr }}$ [7]. Cette démonstration est inspirée de la loi exprimant la densité de courant d'échange d'un processus d'oxydo-réduction à son potentiel d'équilibre selon Butler-Volmer. Si les réactions anodique et cathodique suivent le processus d'activation exprimé par la loi de Tafel [7], la densité de courant global $j$ autour du potentiel de corrosion peut être exprimée par:

$$
j=j_{\text {corr }} \cdot\left[\exp \left(b_{\mathrm{a}} \cdot \eta\right)-\exp \left(b_{\mathrm{c}} \cdot \eta\right)\right] .
$$

Ici, $b_{\mathrm{a}}$ et $b_{\mathrm{c}}$ sont respectivement les coefficients de Tafel anodique et cathodique et $\eta$ la différence de potentiel par rapport au potentiel de corrosion (ou circuit ouvert) $E_{\text {corr }}$.

Alors, si la validité de l'équation (3) est vérifiée, la densité de courant anodique $j_{\mathrm{a}}$ et cathodique $j_{c}$, à des potentiels suffisamment éloignés de $E_{\text {corr }}$, aboutissent à une droite de Tafel $\log (|j|)-E$. L'intersection de ces deux droites permet d'évaluer la densité de courant de corrosion $j_{\text {corr }}$. Pour les mêmes raisons le potentiel ainsi obtenu doit coïncider avec celui déterminé expérimentalement en circuit ouvert. Or, très souvent ce n'est pas le cas. Une des raisons de cet écart est la présence de cellules locales qui, comme l'ont introduit Wagner et Traud [4] produit une partition de la surface.

Supposons que les réactions anodique et cathodique se déroulent respectivement sur une fraction de la surface de l'électrode $\theta_{\mathrm{a}}$ et $\theta_{c}$ et que pour chacune de ces aires de surface, la réaction suit la loi de Tafel, alors nous pouvons réécrire l'équation (3) comme :

$$
\begin{aligned}
& j=j_{\mathrm{a}} \cdot \theta_{\mathrm{a}} \cdot \exp \left(b_{\mathrm{a}} \cdot \eta\right)-j_{\mathrm{c}} \cdot \theta_{\mathrm{c}} \cdot \exp \left(b_{\mathrm{c}} \cdot \eta\right) \\
& j_{\text {corr }}=j_{\mathrm{a}} \cdot \theta_{\mathrm{a}, \text { corr }}=-j_{\mathrm{c}} \cdot \theta_{\mathrm{c}, \text { corr }} .
\end{aligned}
$$



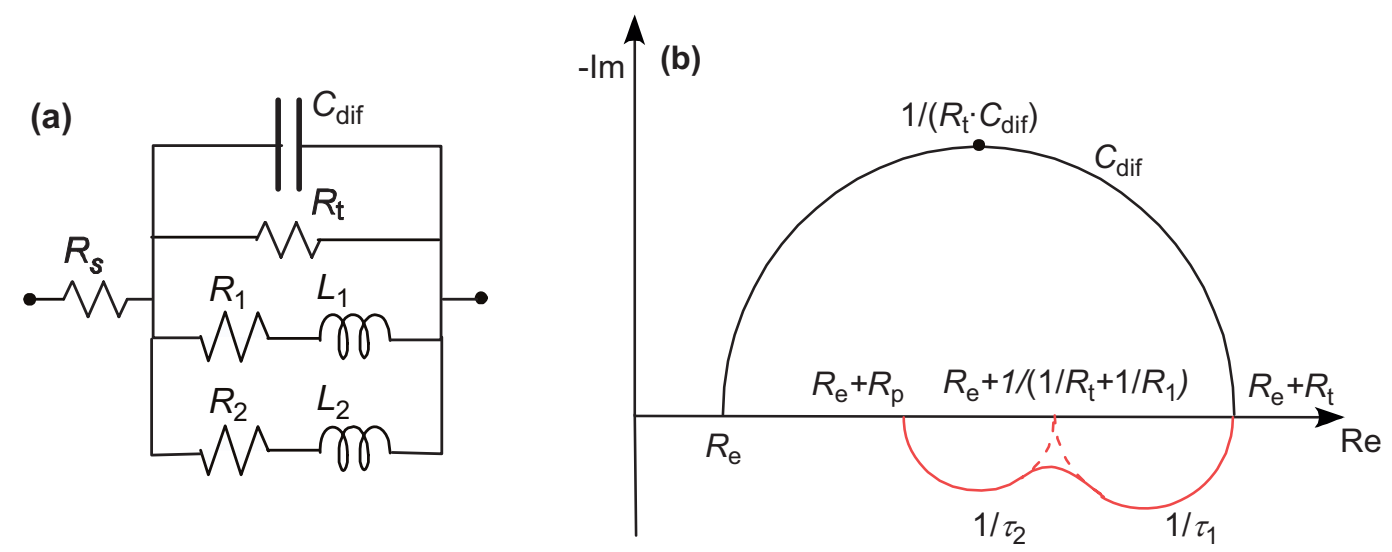

Fig. 2. (a) Circuit électrique équivalent du comportement dynamique de l'interface électrochimique avec deux constantes de temps inductives. (b) Diagramme de Nyquist correspondant $1 / \rho=1 / R_{\mathrm{t}}+1 / R_{1}$ et $1 / R_{\mathrm{p}}=1 / R_{\mathrm{t}}+1 / R_{1}+1 / R_{2}$.

Fig. 2. (a) Equivalent electrical circuit of the dynamic behaviour of the electrode interface with two time constants under inductive feature. (b) Nyquist diagram corresponding to $1 / \rho=1 / R_{t}+1 / R_{1}$ and $1 / R_{p}=1 / R_{t}+1 / R_{1}+1 / R_{2}$.

Tableau 1. La relation de Stern-Geary étendue à la résistance de transfert de charge $R_{\mathrm{t}}$ pour différents types de réaction.

Table 1. The comparison of Stern-Geary relationship with the polarization resistance $R_{p}$ and that extended to the charge transfer resistance $R_{t}$ for different reaction types.

\begin{tabular}{|l|l|l|l|l|}
\hline & \multicolumn{2}{|l|}{ Type de réaction } & $B$ & $B^{\prime}$ \\
\hline Cas & Anodique & Cathodique & $1 / j_{\text {corr }} \cdot R_{\mathrm{p}}$ & $1 / j_{\text {corr }} \cdot R_{\mathrm{t}}$ \\
\hline 1 & $\mathrm{~A} \rightarrow \mathrm{B}$ & $\mathrm{C} \rightarrow \mathrm{D}$ & $b_{\mathrm{a}}-b_{\mathrm{c}}$ & $b_{\mathrm{a}}-b_{\mathrm{c}}$ \\
\hline 2 & $\mathrm{~A} \rightarrow \mathrm{B}$ & $\mathrm{C} \rightarrow \mathrm{D}$ & $b_{\mathrm{a}}$ & $b_{\mathrm{a}}-b_{\mathrm{c}}$ \\
\hline 3 & $\mathrm{~A} \rightarrow \mathrm{B}$ & $\mathrm{C} \rightarrow \mathrm{D}$ & $?$ & $b_{\mathrm{a}}-b_{\mathrm{c}}$ \\
\hline 4 & $\mathrm{~A} \rightarrow \mathrm{A}^{\prime} \ldots \rightarrow \mathrm{B}$ & $\mathrm{C} \rightarrow \mathrm{C}^{\prime} \ldots \rightarrow \mathrm{D}$ & $?$ & $b_{\mathrm{a}}-b_{\mathrm{c}}$ \\
\hline 5 & $\mathrm{~A} \Leftrightarrow \mathrm{A}^{\prime} \rightarrow \mathrm{B}$ & $\mathrm{C} \rightarrow \mathrm{D}$ & $-b_{\mathrm{c}}$ & $?$ \\
\hline
\end{tabular}

$\rightarrow$ : processus contrôlé par activation,

$\Rightarrow$ : processus limité par diffusion,

$\rightarrow$ : processus partiellement contrôlé par diffusion (cinétique mixe),

cas 5 : corrosion des métaux passifs,

? : expression dépendant des conditions expérimentales,

$b_{\mathrm{a}}, b_{\mathrm{c}}$ : moyenne arithmétique de $b_{\mathrm{a}}$ et $b_{\mathrm{c}}$ pour des processus multi-étapes consécutives.

$\theta_{\mathrm{a}}$ et $\theta_{\mathrm{c}}$ dépendent du potentiel et leur valeur à $E_{\text {corr }}$ est représentée par l'indice " corr ". La méthode d'extrapolation de la droite de Tafel n'est valable que si $j_{\mathrm{a}} \cdot \theta_{\mathrm{a}}$ et $j_{\mathrm{c}} \cdot \theta_{\mathrm{c}}$ suivent la loi de Tafel dans un large domaine de potentiel, ce qui implique que $\theta_{\mathrm{a}}$ et $\theta_{\mathrm{c}}$ sont indépendants du potentiel, ou bien que l'un des deux paramètres, par exemple $\theta_{c}$, est proche de l'unité au potentiel du circuit ouvert. Dans ce cas, l'extrapolation de la branche cathodique jusqu'à $E_{\text {corr }}$ déterminé expérimentalement permet d'évaluer $j_{\text {corr }}$ avec une précision suffisante pour l'étude de la corrosion.

À partir de l'équation (3) nous obtenons :

$$
\begin{aligned}
\left.\frac{\mathrm{d} j}{\mathrm{~d} E}\right|_{E_{\text {corr }}=} & \frac{1}{R_{\mathrm{p}}}=j_{\text {corr }} \cdot\left[b_{\mathrm{a}} \cdot \exp \left(b_{\mathrm{a}} \cdot \eta\right)\right. \\
& \left.-b_{\mathrm{c}} \cdot \exp \left(b_{\mathrm{c}} \cdot \eta\right)\right]=j_{\text {corr }} \cdot\left(b_{\mathrm{a}}-b_{\mathrm{c}}\right) .
\end{aligned}
$$

C'est cette équation que Stern et Geary ont introduite pour l'évaluation de $j_{\text {corr }}$. En effet, nous pouvons réécrire aisément, d'après l'équation (5) que :

$$
j_{\text {corr }}=\frac{1}{R_{\mathrm{p}}} \cdot \frac{1}{b_{\mathrm{a}}-b_{\mathrm{c}}}=\frac{B}{R_{\mathrm{p}}} \text {. }
$$

Dans la majorité des cas $1 /\left(b_{a}-b_{c}\right)$ vaut environ $20 \mathrm{mV}$.

Si nous tenons compte des cellules locales, d'après l'équation (4), nous pouvons écrire :

$\frac{d I}{d E}=\frac{1}{R_{p}}=I_{\text {corr }}\left(b_{a}-b_{c}\right)+\frac{1}{\theta_{a, \text { corr }}} \frac{d \theta_{a}}{d E}+\frac{1}{\theta_{c, \text { corr }}} \frac{d \theta_{c}}{d E}$.

Si $\theta_{\mathrm{a}}$ et $\theta_{\mathrm{c}}$ sont indépendants du potentiel, alors les équations $(6,7)$ se confondent, et nous pouvons appliquer la relation de Stern-Geary.

\section{Impédance faradique}

Les variations de $\theta_{\mathrm{a}}$ et $\theta_{\mathrm{c}}$ sous l'effet d'un changement de potentiel sont cinétiquement 
Tableau 2. Manifestation de l'impédance faradique.

Table 2. Faradic impedance feature from the electrochemical kinetics point of view.

\begin{tabular}{|l|l|l|}
\hline & $g_{E}^{\prime}<0$ & $g_{E}^{\prime}<0$ \\
\hline$\partial f / \partial X \quad>0$ & Adsorption de catalyse (Inductif) & Désorption de catalyse(Capacitif) \\
\hline$\partial f / \partial X<0$ & Adsorption d'inhibiteur(Capacitif) & Désorption d'inhibiteur(Inductif) \\
\hline
\end{tabular}

contrôlées et ne peuvent pas être instantanées, une constante de temps de relaxation $\tau$ apparaît dans la variation $\mathrm{de} \mathrm{d} \theta / \mathrm{d} E$. Sous le régime de perturbation sinusoïdale à une pulsation $\omega(=2 \cdot \pi \cdot f, f$ en $\mathrm{Hz})$, nous pouvons écrire :

$$
\frac{\mathrm{d} \theta}{\mathrm{d} E}=\left(\frac{\mathrm{d} \theta}{\mathrm{d} E}\right)_{E_{\mathrm{s}, j_{\mathrm{s}}}} \cdot\left(\frac{1}{1+j \cdot \omega \cdot t}\right)
$$

Pour des fréquences suffisamment élevées $(\omega \cdot \tau>>1)$, le terme $\mathrm{d} \theta / \mathrm{d} E$ est égal à zéro, la relation de Stern-Geary devient valide même si dans l'état stationnaire $\theta_{\mathrm{a}}$ et $\theta_{\mathrm{c}}$ dépendent de $E$. La méthode d'impédance repose sur ce principe.

$$
\begin{aligned}
\frac{1}{Z_{\mathrm{F}}}=\frac{\mathrm{d}_{j}}{\mathrm{~d}_{E}}(\omega)= & \frac{1}{R_{t}}+\frac{1}{R_{1}} \cdot \frac{1}{1+j \omega \cdot \tau_{1}} \\
& +\frac{1}{R_{2}} \cdot \frac{1}{1+j \omega \cdot \tau_{2}} .
\end{aligned}
$$

La résistance de transfert de charge $R_{\mathrm{t}}$ représente la réponse de l'interface à une fréquence suffisamment élevée, $\omega<<1 / \tau$, de telle sorte qu'aucun phénomène de relaxation n'ait lieu. Comme phénomènes impliqués dans les processus de corrosion, nous pouvons citer le taux de recouvrement de surface par des intermédiaires de réaction, la concentration à l'interface des espèces réagissantes, l'adsorption d'inhibiteurs. . . Ceci donne un large éventail de situations où $R_{\mathrm{t}}$ est directement reliée à la vitesse de corrosion, $j_{\text {corr }}$.

L'interface électrochimique est composée, en plus de l'impédance faradique $Z_{F}$ (Éq. (9)), de la résistance d'électrolyte $R_{\mathrm{e}}$ et de la capacité de double couche $C_{\text {dif }}$ comme nous l'avons illustré sur la figure 1 . Nous pouvons alors le représenter par le circuit électrique équivalent et par le diagramme de Nyquist correspondant (Fig. 2).

Le tableau 1 résume la relation entre la vitesse de corrosion et les deux grandeurs résistives, $R_{\mathrm{t}}$ ou $R_{\mathrm{p}}$.

Sur ce tableau, nous pouvons constater que la relation de Stern-Geary s'applique dans beaucoup de situations cinétiques avec la résistance de transfert de charge $R_{\mathrm{t}}$ plutôt qu'avec la résistance de polarisation $R_{\mathrm{p}}$. La résistance de transfert de charge doit donc être utilisée plutôt que la résistance de polarisation. Toutefois, pour un système où un processus rédox rapide se déroule eu même temps que le processus de corrosion, c'est $R_{\mathrm{p}}$ qui est sera plus étroitement associée à $j_{\text {corr }}$.

\section{Impédance faradique capacitive ou inductance}

Le courant traversant l'interface électrochimique dépend de façon générale du potentiel $E$ et d'une variable arbitraire $X$ :

$$
j=f(E, X) .
$$

Le développement de Taylor de cette équation, limité au premier ordre donne :

$$
\Delta j=\left(\frac{\partial f}{\partial E}\right)_{\mathrm{X}_{\mathrm{s}}, \mathrm{E}_{\mathrm{s}}} \cdot \Delta E+\left(\frac{\partial f}{\partial X}\right)_{\mathrm{X}_{\mathrm{s}}, \mathrm{E}_{\mathrm{s}}} \cdot \frac{\Delta X}{\Delta E}
$$

Nous pouvons alors calculer l'impédance faradique par :

$$
\begin{aligned}
& \frac{1}{Z_{\mathrm{F}}}=\frac{\Delta j}{\Delta E}=\left(\frac{\partial f}{\partial E}\right)_{\mathrm{X}_{\mathrm{s}}, \mathrm{E}_{\mathrm{s}}}+\left(\frac{\partial f}{\partial X}\right)_{\mathrm{X}_{\mathrm{s}}, \mathrm{E}_{\mathrm{s}}} \cdot \frac{\Delta X}{\Delta E} \\
& =\frac{1}{R_{\mathrm{t}}}+\left(\frac{\partial f}{\partial \mathrm{X}}\right)_{\mathrm{X}_{\mathrm{s}}, \mathrm{E}_{\mathrm{s}}} \cdot \frac{g_{\mathrm{E}}}{\mathrm{j} \cdot \omega-g_{\mathrm{X}}^{\prime}} .
\end{aligned}
$$

L'équation (12) montre que l'impédance faradique est constituée de deux termes :

- à $\omega=\infty, Z_{\mathrm{F}}=R_{\mathrm{t}}$, c'est-à-dire la résistance de transfert de charge lorsque $X$ ne peut pas suivre la perturbation du potentiel $\Delta E$.

- à $\omega=0, Z_{\mathrm{F}}$ est définie comme résistance de polarisation $R_{\mathrm{p}}$.

Pour que le système soit stable, la constante du temps du système $\tau>0$ donc $g^{\prime}$ x négatif. Puisque le phénomène de relaxation dans l'impédance est déterminé par le signe de :

$$
\left(\frac{\partial f}{\partial X}\right)_{\mathrm{X}_{\mathrm{s}}, \mathrm{E}_{\mathrm{s}}} \cdot g_{\mathrm{E}}^{\prime}
$$

Alors quatre cas peuvent être considérés : si le produit est positif, l'impédance est inductive, dans le cas contraire, elle est capacitive. Schématiquement, on peut les présenter par le tableau 2. 


\section{Résultats et discussion}

Dans la grande majorité des cas, l'application de la résistance de transfert à l'estimation de la vitesse de corrosion donne des résultats satisfaisants. Nous illustrons ici des cas pathologiques où une application directe de la S.I.E. pour l'évaluation de la vitesse de corrosion est plus ou moins compromise.

\section{Résistance d'électrolyte}

Le calcul des distributions de courant et de potentiel dans une cellule électrochimique, dans la phase électrolytique, permet de déterminer la résistance d'électrolyte. Pour une cellule de forme quelconque, une technique numérique, comme la méthode des éléments finis, évaluera pour chaque cas particulier la valeur de $R_{\mathrm{e}}$. En revanche, pour des géométries favorables on dispose de solutions analytiques : pour une électrode à disque de rayon $a$ dans un plan de rayon infini placée dans une cellule hémisphérique également de rayon infini dans une solution de résistivité $\rho$, Newman a calculé la distribution du potentiel et les lignes de courant (orthogonales au équipotentielles), puis la résistance d'électrolyte [8].

$$
R_{\mathrm{e}}=\frac{\rho}{4 \cdot a} .
$$

C'est une expression importante car parfois les artefacts instrumentaux introduisent des spectres parasites, notamment à hautes fréquences, rendant l'interprétation des résultats difficile.

\section{Milieu fortement résistif}

Lors de la synthèse de l'acide acétique anhydre, dit glacial, la conductivité du milieu baisse énormément lorsque la teneur en eau libre diminue. Le diagramme d'impédance obtenu est présenté sur la figure 3 [9].

Quand l'électrode de référence est située sur l'axe de l'électrode de travail cylindrique, il est possible d'évaluer la résistance d'électrolyte à la distance de $z$ [8] :

$$
R_{\mathrm{e}}=\left(\frac{\rho}{4 \cdot a}\right) \cdot\left(\frac{2}{\pi}\right) \cdot \tan ^{-1}\left(\frac{z}{a}\right) .
$$

Avec $\rho=2,5 \mathrm{M} \Omega \mathrm{cm}, a=0,56 \mathrm{~cm}$, nous constatons que $R_{\mathrm{e}}$ s'identifie avec le point où le spectre d'impédance coupe l'axe des réels vers $1 \mathrm{~Hz}$. (Cette valeur de $\rho$ est inférieure à celle de l'acide acétique anhydre,

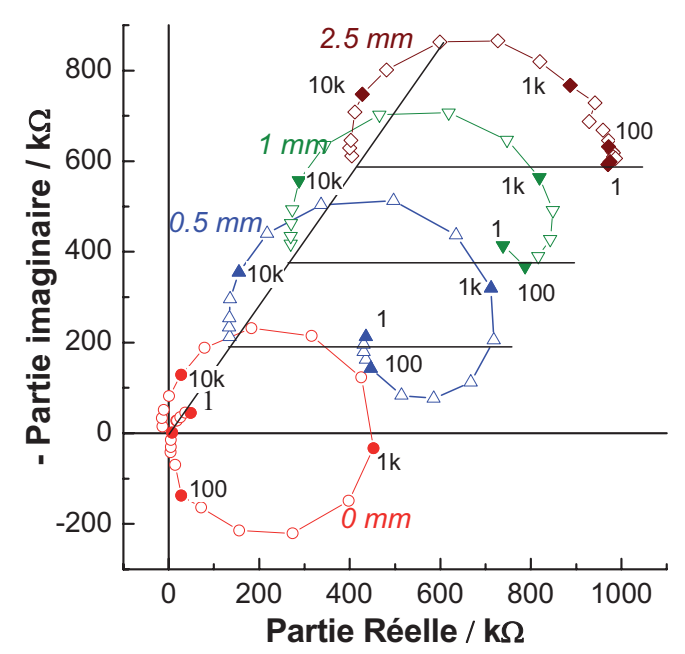

Fig. 3. Spectres d'impédance obtenus sur un acier au carbone dans $\mathrm{CH}_{3} \mathrm{COOH}$ quasianhydre pour différentes valeurs de position de l'électrode de référence.

Fig. 3. Impedance spectra obtained at the carbon steel electrode immersed in quasi-anhydrous $\mathrm{CH}_{3} \mathrm{COOH}$ for different position of the reference electrode capillary tip.

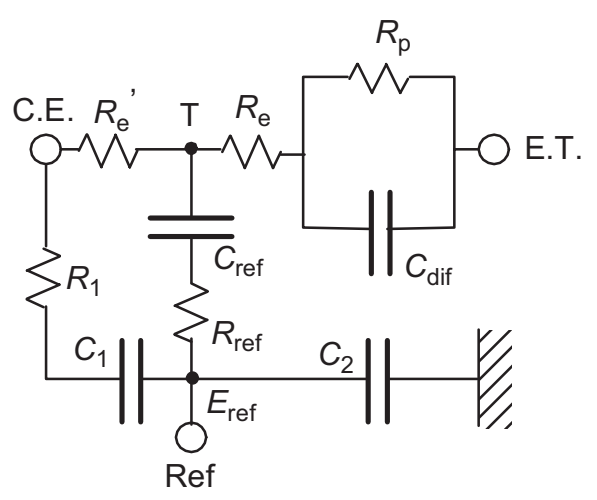

Fig. 4. Schéma électrique équivalent décrivant l'artéfact de mesure : C.E : contre électrode ; E.T. électrode de travail, Ref : entrée électrode de référence de potentiostat.

Fig. 4. Schematic representation in electrical equivalent circuit of the electrochemical cell including regulating device: C.E. : counter electrode; E.T. working electrode, Ref: potentiostat input of the reference electrode.

dans les conditions expérimentales utilisées, des traces d'eau devraient rester encore dans l'acide). Pour des fréquences inférieures, apparaît l'amorce d'une boucle capacitive. La capacité parallèle observée correspond, en plus, aux valeurs fréquemment rencontrées pour $C_{\text {dif }}$. Cette réponse, pur artefact de mesure, a été expliquée par les caractéristiques électriques de l'électrode de référence et de l'impédance d'entrée du potentiostat vue par cette électrode (Fig. 4). 


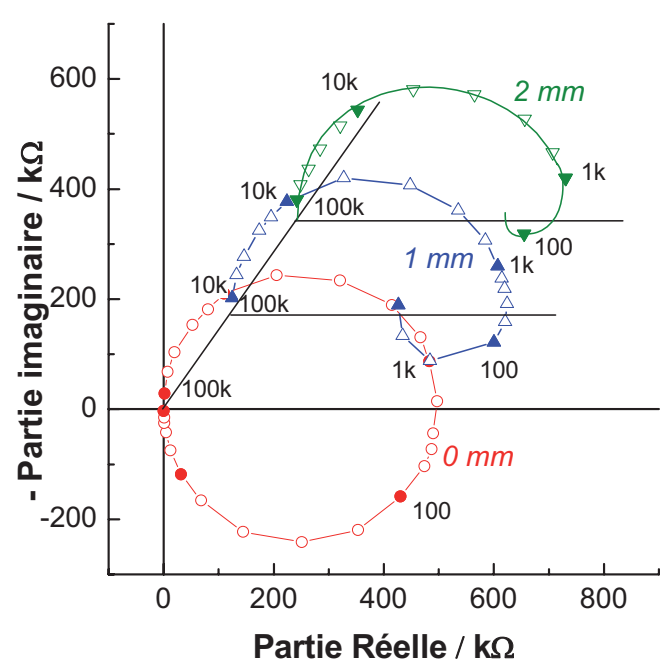

Fig. 5. Impédance calculée avec le circuit électrique équivalent de la figure $4: \rho=2.5 \mathrm{M} \Omega \mathrm{cm}$; couplage Ref -C.E. : $R_{1}=2 \mathrm{M} \Omega, C_{1}=10 \mathrm{pF} ; \mathrm{Ag}$ | AgCl + capillaire : $R_{\text {ref }}=100 \mathrm{M} \Omega, C_{\text {ref }}=1 \mathrm{MF}$ (pas de contribution de cette capacité); capacité parasite P-stat : $C_{2}=30 \mathrm{pF}$; E.T. : $R_{\mathrm{p}}=500 \mathrm{k} \Omega$, $C_{\mathrm{d}}=\mathbf{1 0 0} \mu \mathrm{F}$.

Fig. 5. Calculated impedance with electrical equivalent circuit presented in Figure 4: $\rho=2.5 \mathrm{M} \Omega \mathrm{cm}$; Coupling of Ref-C.E.: $R_{1}=2 \mathrm{M} \Omega, C_{1}=10 \mathrm{pF}$; $\mathrm{Ag} \mid \mathrm{AgCl}+$ Luggin capillary: $R_{\text {ref }}=100 \mathrm{M} \Omega$, $C_{\text {ref }}=1 \mathrm{MF}$ (no contribution of the capacitance); parasitic capacitance of P-stat: $C_{2}=30 \mathrm{pF}$; E.T.: $R_{p}=500 \mathrm{k} \Omega, C_{d}=100 \mu \mathrm{F}$.

L'extrémité de l'électrode de référence (position $\mathrm{T}$ ) partage la résistance d'électrolyte $\left(R_{\text {tot }}\right)$ en deux : une partie du côté de la contre-électrode $\left(R_{\mathrm{e}}^{\prime}\right)$ et l'autre du côté de l'électrode de travail $\left(R_{\mathrm{e}}\right) . R_{\text {ref }}-C_{\text {Ref }}$ représente l'impédance de l'électrode de référence avec l'embout en capillaire de Luggin, $C_{2}$, la capacité parasite de l'impédance d'entrée de la référence sur le circuit du potentiostat et $R_{\mathrm{p}}-C_{\mathrm{dif}}$ représente l'impédance de l'électrode d'acier immergée dans l'acide acétique.

Avec ce modèle, nous avons pu reproduire, comme le montre la figure 5, les spectres d'impédance représentés sur la figure 3 avec des valeurs raisonnables pour les différentes variables géométriques et physicochimiques [9]. Ce schéma explique également le bienfait d'un fil de platine accolé à l'électrode de référence pour diminuer l'artefact observé, par la diminution de la valeur de $R_{\text {ref }}$.

\section{Capacité différentielle de double couche}

Des alcools acétyléniques comme l'alcool propargylique (A.P.) ou le butyne-diol sont connus pour leur effet inhibiteur en milieu acide. La figure $6 a$ montre deux spectres d'impédance obtenus en absence et en présence de 0,5 mM A.P. En présence de l'inhibiteur, nous observons deux boucles inductives en basses fréquences comme nous $l^{\prime}$ avons illustré sur la figure $2 \mathrm{~b}$. Une augmentation significative du module d'impédance en présence de l'A.P. observée indique un effet inhibiteur notable [10].

La figure $6 \mathrm{~b}$ montre la comparaison $\mathrm{du}$ pouvoir inhibiteur $(H)$ évalué par différentes méthodes, L'efficacité inhibitrice est calculée par l'équation suivante.

$$
H(\%)=100 \cdot \frac{V_{0}-V}{V_{0}}
$$

$V_{0}$ et $V$ indiquent respectivement la vitesse de corrosion en absence et en présence d'inhibiteur. La mesure directe par perte de masse $\left(H_{\mathrm{m}}\right)$ sert de référence. On constate que le pouvoir inhibiteur atteint plus de $90 \%$, et la résistance de transfert de charge indique une estimation de l'effet inhibiteur $\left(H_{R_{t}}\right)$ la plus proche à la référence. En revanche, pour des concentrations en A.P. supérieures à $2 \mathrm{mM}, R_{\mathrm{p}}$ en présence de l'inhibiteur devient plus petit qu'en son absence, alors $H_{R_{p}}$ devient négative en complet désaccord avec la mesure directe.

\section{Modulation de la capacité de double couche pendant la mesure d'impédance}

Généralement, nous admettons que $C_{\text {dif }}$ est constante dans tout le domaine de fréquence, ou suit une loi de puissance (CPE $\left.\left(C_{\text {dif }}\right)=Q \cdot(j \cdot \omega)^{n}\right)$. Cette capacité est complètement dissociée du processus faradique. Or, on sait également que la capacité de double couche varie avec le potentiel comme le prévoient les modèles de Gouy-ChapmanStern pour une électrode idéalement polarisable. Cette capacité dépend également des réactions électrochimiques en raison de la modification de la structure de la double couche sous l'effet du flux de charges et de la présence de phases 2-D ou 3-D à l'interface. La variation de capacité sous l'effet du régime sinusoïdal est déterminée expérimentalement par superposition des deux signaux alternatifs ; fréquence variable pour la mesure traditionnelle de $Z$ et fréquence de $1 \mathrm{kHz}$ pour extraire la réponse de $C_{\text {dif }}$ [11].

Nous présentons les résultats de mesure de $Z$ dans le plan d'admittance $(1 / Z)$, les courbes (a) et (c) montrent dans les deux 

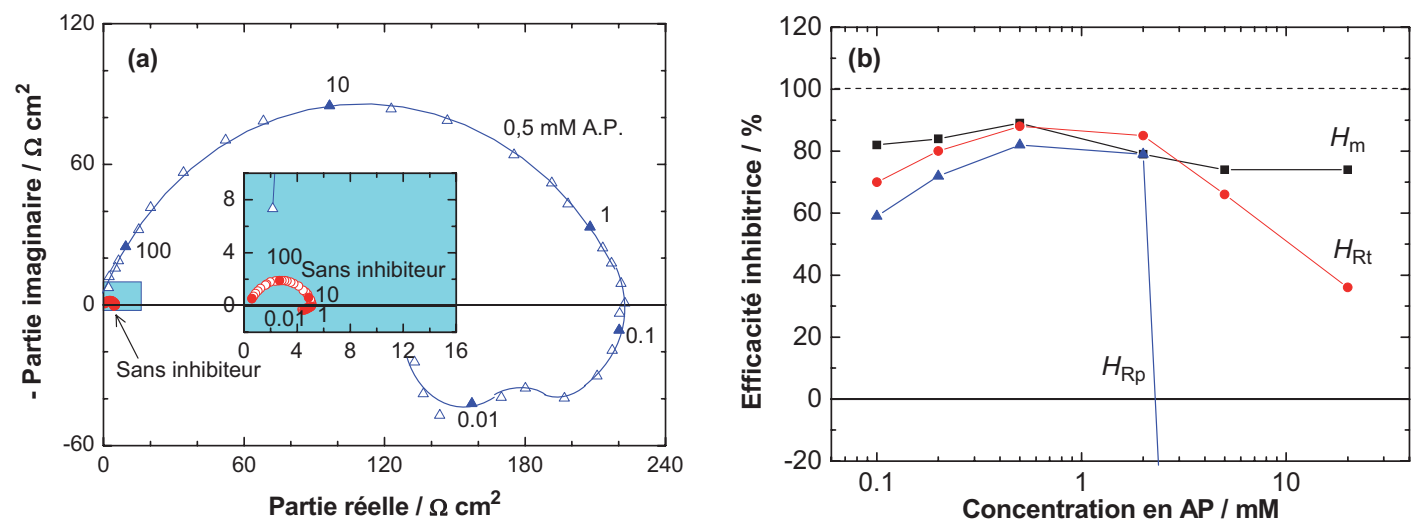

Fig. 6. Fe pur industriel | $1 \mathrm{M} \mathrm{H}_{2} \mathrm{SO}_{4}$; (a) impédance en absence et en présence de l'inhibiteur. (b) Comparaison du pouvoir inhibiteur évalué par différentes méthodes. $H_{\mathrm{m}}=$ mesure de perte de masse, $H_{\mathrm{Rt}}=$ résistance de transfert de charge $; H_{\mathrm{Rp}}=$ résistance de polarisation.

Fig. 6. Pure industrial $\mathrm{Fe} \mid 1 \mathrm{M} \mathrm{H}_{2} \mathrm{SO}_{4}$; (a) impedance in absence and in presence of the inhibitor; (b) comparison of inhibiting efficiency evaluated from various methods. $H_{\mathrm{m}}=$ mass loss, $H_{\mathrm{Rt}}=$ charge transfer resistance; $H_{\mathrm{Rp}}=$ polarisation resistance measurements.
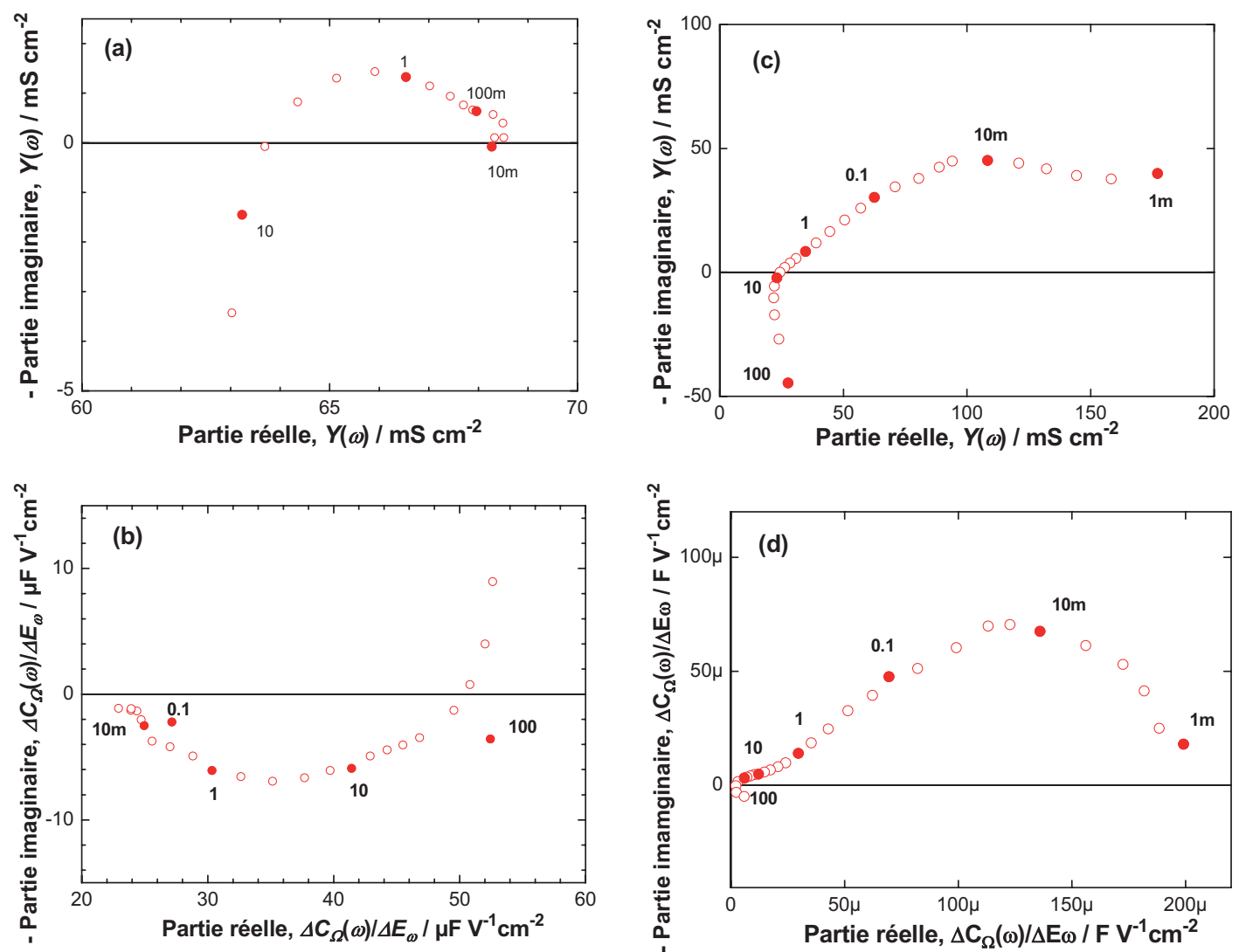

Fig. 7. Les spectres en admittance (a, b) et en réponse en $\mathrm{Cd}(\mathrm{c}, \mathrm{d}) \mathrm{Fe} \mid \mathbf{1} \mathrm{M} \mathrm{H}_{2} \mathrm{SO}_{4}(\mathrm{a}, \mathrm{b})$ sans inhibiteur $(c, d) 1 \mathrm{mM}$ alcool propargylique.

Fig. 7. Admittance spectra (1/Z) (a, c) and the $\Delta \mathrm{C}_{\Omega}$ response $(b, d) \mathrm{Fe} \mid 1 \mathrm{M} \mathrm{H}_{2} \mathrm{SO}_{4}(a, b)$ No inhibitor (c, d) 1 mM propargylic alcohol.

milieux un comportement inductif. La variation de $C_{\text {dif }}$ est au contraire très différente entre les deux cas (voir Fig. 7). La capacité diminue quand la fréquence décroît en absence de l'inhibiteur alors qu'elle augmente en sa présence. En absence d'inhibiteur, l'espèce intermédiaire de réaction généralement considérée est $\mathrm{Fe}(\mathrm{OH})$.
La désorption anodique de l'inhibiteur, sans doute associé à $H_{\text {ads }}$, fixé sur le substrat de fer [12] fait, au contraire, augmenter la valeur de $C_{\text {dif. }}$. Ce résultat illustre clairement que la mesure de $C_{\text {dif }}$ en fonction de la fréquence permet de distinguer clairement la nature de l'adsorbat et son rôle cinétique alors que $Z_{\mathrm{F}}$ ne peut pas de lever l'ambiguïté 


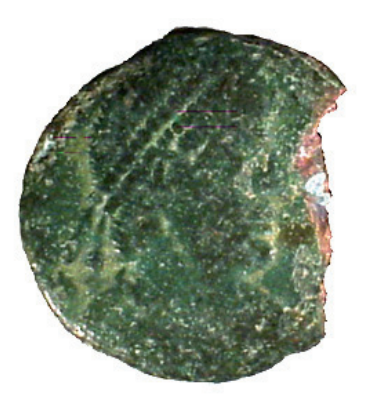

(a)

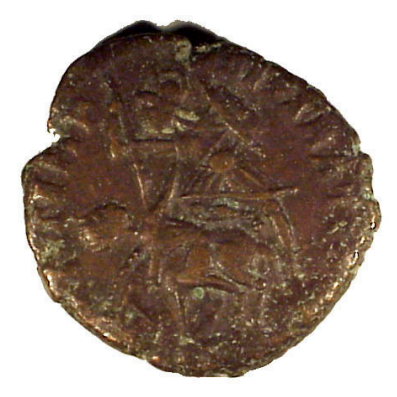

(b)

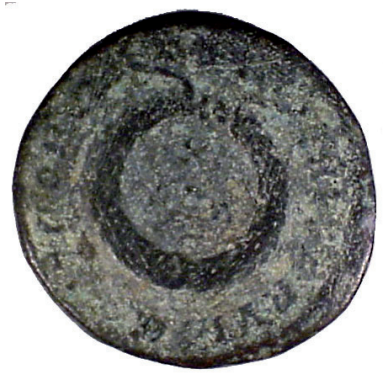

(c)

Fig. 8. Clichés de quelques pièces de monnaie du Bas Empire Romain. Les diamètres de ces pièces sont toutes environ $15 \mathrm{~mm}$.

Fig. 8. Pictures of some coins dated of the post Roman Empire. The diameter of these coins are all of them ca. $15 \mathrm{~mm}$.

entre l'adsorption de l'espèce catalytique et la désorption d'inhibiteur comme nous l'avons illustré sur le tableau 2.

\section{Protection des pièces de monnaie en bronze}

Les pièces de monnaie examinées proviennent du Maroc et sont datables du BasEmpire Romain (IV siècle après JC). Leurs formes d'origine n'ont pas changé même si leurs inscriptions sont devenues difficilement déchiffrables sur les pièces souvent recouvertes de patine due au phénomène de corrosion. La figure 8 montre trois pièces de monnaie utilisées dans cette étude [13].

Sur une des pièces (a), nous avons prélevé une petite partie par découpe et l'avons enrobée dans une résine époxy. La surface est polie au papier émeri jusqu'au ${ }^{\circ} 1200$. Pour l'observation par M.E.B., un polissage supplémentaire à l'alumine est effectué. Avant de rechercher les caractéristiques électrochimiques du bronze recouvert de patine, les identifications chimiques de celle-ci sont réalisées par l'E.D.S. (Energy Dispersive Xray Spectroscopy) et la micro-spectroscopie Raman. Le tableau 3 résume le résultat d'analyse. Ce tableau indique que la pièce examinée contient une quantité importante $\mathrm{de} \mathrm{Pb}$ comme une autre pièce de monnaie archéologique marocaine analysée auparavant [13].

L'analyse Raman sur la patine formée sur la pièce ' $a$ ' (patine verte) a montré la présence de $\mathrm{Cu}_{2} \mathrm{O}$ (cuprite) et de $\mathrm{SnO}_{2}$ (cassiterite) à la surface alors que la partie interne de la patine révèle la présence de $\mathrm{Cu}_{2} \mathrm{O}$ et de la couche passive native du cuivre $\left(500-620 \mathrm{~cm}^{-1}\right)$. Nous avons également constaté que dans la patine, le $\mathrm{Pb}$ se trouve sous forme $\alpha$ - $\mathrm{PbO}$ (litharge :
Tableau 3. Composition de la pièce en bronze 1a. Table 3. Elemental composition of the bronze coin $1 a$.

\begin{tabular}{|l|l|l|l|l|l|}
\hline & $\mathrm{Cu}$ & $\mathrm{Sn}$ & $\mathrm{Pb}$ & $\mathrm{Fe}$ & $\mathrm{Al}$ \\
\hline$\%$-at & 80,0 & 7,75 & 10,90 & 0,74 & 0,54 \\
\hline$\%$-mas & 61,1 & 11,1 & 27,2 & 0,24 & 0,36 \\
\hline
\end{tabular}

$\left.216 \mathrm{~cm}^{-1}\right)$. Dans la matrice de bronze, dans les zones proches de joints de grain, riche en $\mathrm{Pb}$, les spectres Raman indiquent la présence de $\mathrm{Cu}_{2} \mathrm{O}, \mathrm{CuCl} \cdot 2 \mathrm{H}_{2} \mathrm{O}$ (ériochalcite); de $\alpha$ $\mathrm{PbO}, 2 \mathrm{PbCO}_{3} \cdot \mathrm{Pb}(\mathrm{OH})_{2}$ (hydrocérussite), $\mathrm{Pb}-$ $\mathrm{ClOH}$ (lauriaunite), $\mathrm{Pb}_{3}(\mathrm{ClO})_{2}$ (mendipite) et $\mathrm{Cu}_{20} \mathrm{~Pb}_{21} \mathrm{Cl}_{42}(\mathrm{OH})_{40}$ (cumengeite) [14-16].

Nous avons examiné l'effet protecteur de trois composés triazoles contre la dégradation des trois pièces de monnaie recouvertes de patine de couleur différente; verte pour ' $a$ ', brune ' $b$ ' et grise ' $c$ ' [17]. Elles sont immergées pendant une heure dans la solution de $10 \mathrm{mM}$ d'inhibiteur; pièce ' $a$ ' en BTA (Bénzotriazole), pièce ' $b$ ' en BiTA (Bi-Triazole) et pièce ' $c$ ' en ATA (AminoTriazole). Les spectres d'impédances obtenus pour ces échantillons sont présentés sur la figure 9. Une fois le spectre obtenu, les pièces de monnaie sont lavées à l'eau déminéralisée, séchées puis laissées à l'air libre jusqu'à la mesure d'impédance suivante. Avant chaque relevé du spectre, les échantillons sont laissés pendant une heure dans la solution de test de corrosion ne contenant pas d'inhibiteur, $0,2 \mathrm{~g} \mathrm{~L}^{-1} \mathrm{Na}_{2} \mathrm{SO}_{4}+0,2 \mathrm{~g} \mathrm{~L}^{-1}$ $\mathrm{NaHCO}_{3} \mathrm{pH}$ 3. Cette procédure permet de tester l'efficacité de l'inhibiteur d'un objet en bronze exposé par exemple dans une vitrine d'un musée.

Sur les figures, le spectre noté " sans inhibiteur " correspond à celui obtenu avant l'immersion de ces échantillons dans la solution d'inhibiteur. On peut noter que le module d'impédance augmente avec le temps. L'augmentation du module d'impédance 

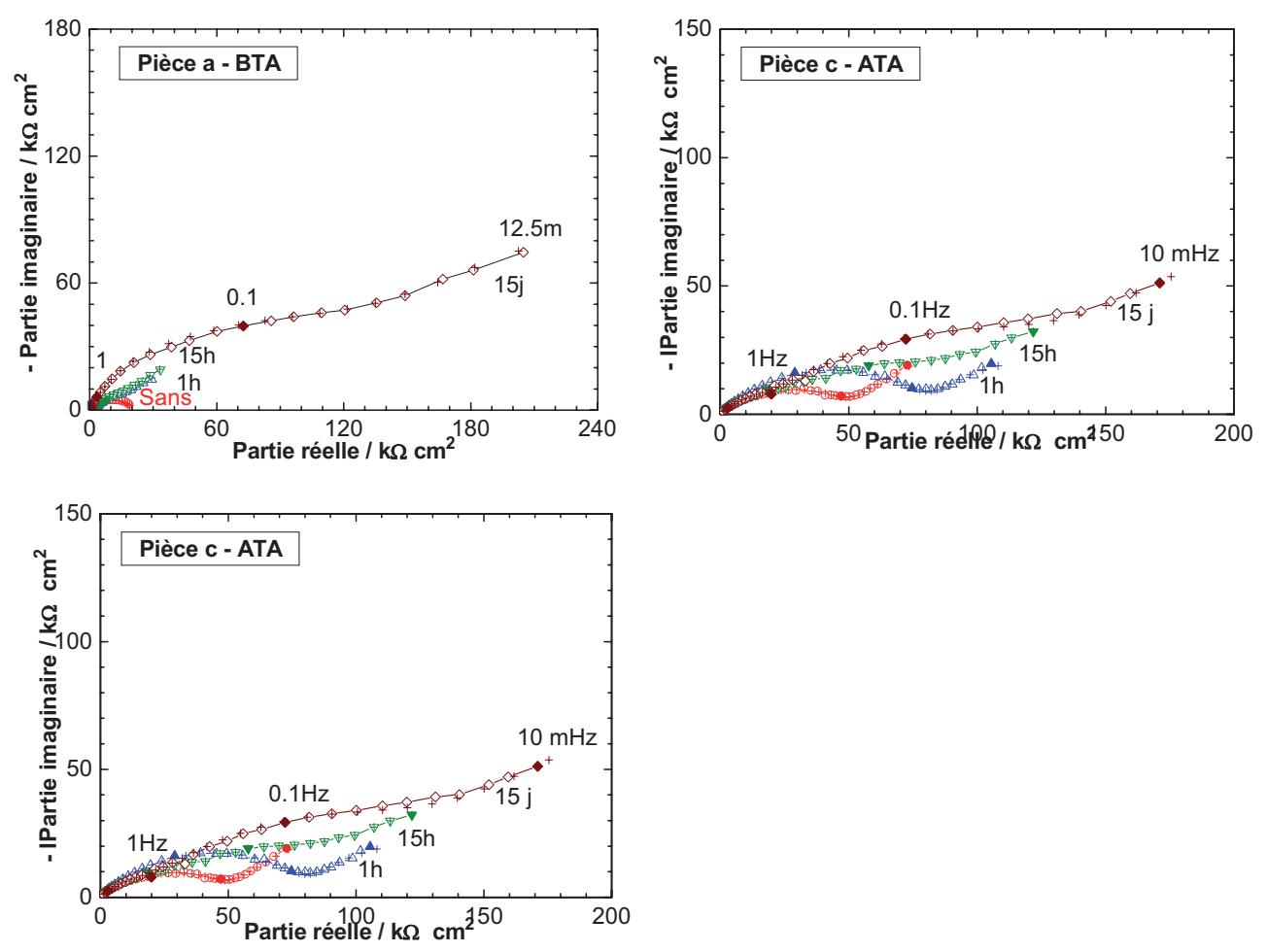

Fig. 9. Évolution du spectre d'impédance des échantillons en exposition à l'air après immersion dans différentes solutions d'inhibiteur $(10 \mathrm{mM})$, puis transfert dans le milieu d'essai de corrosion. $0,2 \mathrm{~g} \mathrm{~L}^{-1} \mathrm{Na}_{2} \mathrm{SO}_{4}+0,2 \mathrm{~g} \mathrm{~L}^{-1} \mathrm{NaHCO}_{3} ; \mathrm{pH} 3$.

Fig. 9. Evolution of the EIS of the coin specimens exposed in air between two measurements. Coins were pre-treaded in a aqueous solution of various inhibitors $(10 \mathrm{mM})$, then transferred to the corrosion test medium: $0.2 \mathrm{~g} \mathrm{~L}^{-1} \mathrm{Na}_{2} \mathrm{SO}_{4}+0.2 \mathrm{~g} \mathrm{~L}^{-1} \mathrm{NaHCO}_{3} ; \mathrm{pH} 3$.

avec le temps est moins marquée pour l'échantillon traitée par BiTA (pièce ' $b$ '). Mais, c'est l'échantillon qui montre le plus grand module d'impédance pour une fréquence donnée.

Le spectre d'impédance présente trois constantes de temps. La boucle capacitive observée à hautes fréquences est attribuée à la couche $d$ 'inhibiteur recouvrant la surface du bronze. En effet en absence d'inhibiteur, l'impédance correspondante à cette boucle est absente. Les inhibiteurs utilisés peuvent en effet former un film polymérique ou complexe avec $\mathrm{Cu}(\mathrm{I})$ ou $\mathrm{Cu}(\mathrm{II})$ [18]. $\mathrm{Ce}$ film présente alors un comportement diélectrique traduit par une capacité $C_{\mathrm{f}}$ en parallèle avec une résistance de conduction ionique $R_{\mathrm{f}}$. La deuxième boucle correspond à la capacité de double couche $C_{\mathrm{d}}$ en parallèle avec la résistance de transfert de charge $R_{\mathrm{t}}$. Enfin, à plus basses fréquences, le processus d'oxydo-réduction de la patine apparaît sous forme de boucle capacitive $\left(C_{\mathrm{F}}-R_{\mathrm{F}}\right)$. La forme très aplatie des deux dernières boucles atteste d'une forte distribution de réactivité électrochimique.
La figure 10 montre la variation avec le temps de $R_{\mathrm{t}}$ et de la résistance de polarisation $R_{\mathrm{p}}\left(=R_{\mathrm{f}}+R_{\mathrm{t}}+R_{\mathrm{F}}\right)$ déterminées à l'aide d'un ajustement de paramètres par une méthode de Simplexe.

En dépit de différences notables dans l'aspect visuel de ces trois pièces de monnaie, la valeur de $R_{\mathrm{t}}$ en absence d'inhibiteur est presque égale à $35 \mathrm{k} \Omega \mathrm{cm}^{2}$ pour ces trois échantillons. Par conséquent nous pensons que la réactivité du substrat de bronze est sensiblement la même pour ces trois pièces de monnaie datant de la même période. Bien que seule la composition d'éléments de la pièce 'a' ait été analysée, elle doit être similaire pour les trois. En effet, cette composition est proche de celle déterminée auparavant [13] sur une autre pièce de monnaie.

Quand les pièces sont traitées par les inhibiteurs, $R_{\mathrm{t}}$ augmente notablement, sauf celle traitée par ATA. Le substrat de bronze est alors bien stabilisé par le traitement de BTA et BiTA. Quant à ATA, comme nous l'avons observé sur l'électrode du $\mathrm{Cu}$, cette substance peut avoir une réactivité électrochimique [18], même si elle se trouve 

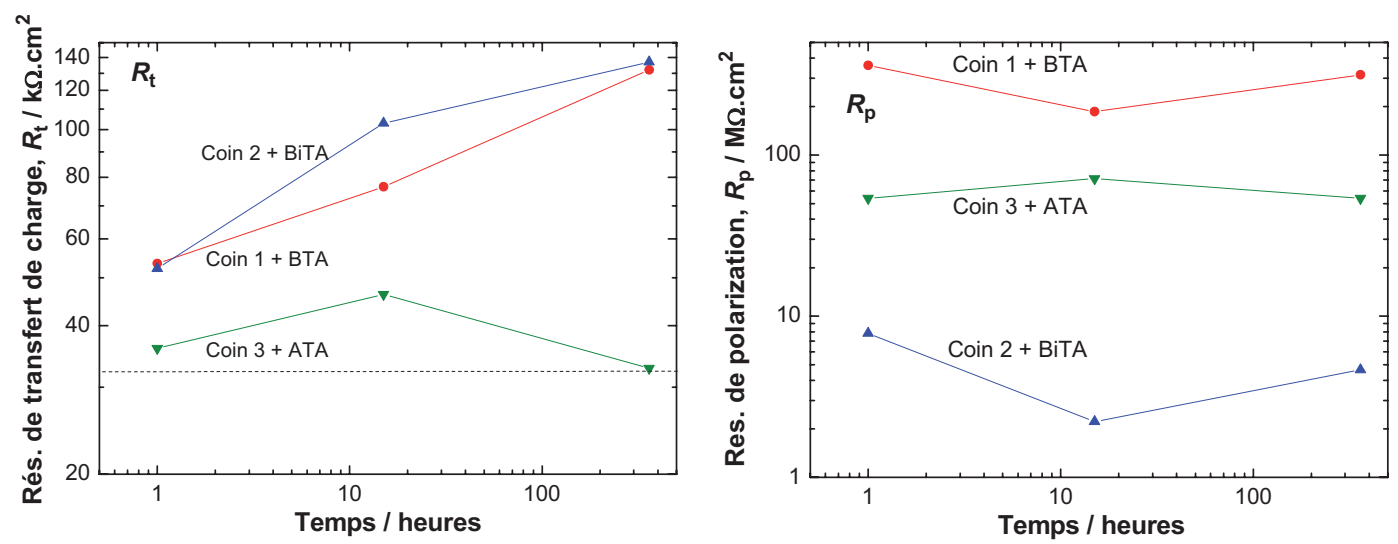

Fig. 10. Évolution de $R_{\mathrm{t}}$ et $R_{\mathrm{p}}$ des pièces de monnaie initialement traitées par trois différents inhibiteurs.

Fig. 10. Admittance spectra (1/Z) $(a, c)$ et and the $\Delta \mathrm{C}_{\Omega}$ response $(b, d) \mathrm{Fe} \mid 1 \mathrm{M} \mathrm{H}_{2} \mathrm{SO}_{4}(a, b)$ no inhibitor $(c, d) 1$ mM propargylic alcohol.

incorporée dans la patine et forme un complexe, au lieu d'être ajoutée dans la solution.

On peut remarquer que le BiTA et le BTA présentent un effet similaire, et le module d'impédance croît même après 15 jours avec immersion intermittente. Puisque l'ATA est électro-actif $R_{\mathrm{t}} \mathrm{n}$ 'indique pas l'efficacité protectrice, la résistance de polarisation $R_{\mathrm{p}}$ est utilisée à cette fin. En effet, en présence d'un processus d'oxydo-réduction, c'est cette résistance qui est la plus étroitement corrélée avec la vitesse de corrosion comme nous l'avons indiqué plus haut (voir Tab. 1, cas 5). La figure $7 \mathrm{~b}$ montre l'évolution de $R_{\mathrm{p}}$ de trois pièces de monnaie archéologique en bronze. Dans ce système, BTA présente la plus grande valeur de $R_{\mathrm{p}}$ ce qui suggère la plus faible vitesse de corrosion. Le traitement de surface par l'ATA est efficace. En revanche, avec le BiTA, nous n'observons pas une augmentation notable de la valeur de $R_{\mathrm{p}}$. Il est cependant important de noter que la valeur de $R_{\mathrm{p}}$ est évaluée au prix d'une large extrapolation du spectre d'impédance, notamment au-delà de 15 heures. Par conséquent les valeurs obtenues ne sont pas entièrement fiables. Néanmoins, une très grande valeur de $R_{\mathrm{p}}$ correspond à une corrosion particulièrement lente. Par exemple si l'on applique la loi relation de Stern-Geary avec le coefficient $B=25 \mathrm{mV}$, comme c'est souvent le cas, $10 \mathrm{M} \Omega \mathrm{cm}^{2}$ correspond à une perte d'épaisseur de moins de $4 \mathrm{~nm}$ par an. La protection contre la corrosion du bronze par les composés triazoles est donc satisfaisante.

Nous avons montré récemment que les autres molécules comme le thiadiazole ou imidazole, dont l'innocuité est vérifiée, protègent également efficacement le bronze patiné [19].

\section{Conclusion}

Depuis un peu plus de 40 ans, l'application de la méthode d'impédance à l'étude de la corrosion a diversifié son champ d'utilisation ; du métal immergé dans un milieu acide à la protection du bronze archéologique par un inhibiteur organique en passant par l'effet d'inhibiteur de corrosion, les aciers recouverts de revêtements organiques ou de nombreux autres problèmes que nous n'avons pas la place d'illustrer ici.

Nous avons montré la différence entre la méthode de Stern et Geary basée sur la mesure de la résistance de polarisation et la mesure de la résistance de transfert de charge. Cette dernière, non seulement est plus rapide à déterminer mais est souvent plus reproductible, elle est moins sensible aux différents processus se déroulant à l'interface métal | électrolyte tels que le recouvrement de surface par des intermédiaires de réaction, l'adsorption-désorption d'inhibiteur, les réactions partiellement ou complètement limitées par la diffusion. . .

Nous avons montré malgré ces avantages indéniables, que la mesure des spectres d'impédance n'est pas à l'abri d'artefacts, par exemple pour un milieu très peu conducteur. L'emploi de l'électrode de référence avec un verre frité, a fortiori, le capillaire de Luggin, peut en effet introduire un spectre purement instrumental, source de gravissimes erreurs d'interprétation.

Nous avons ensuite illustré comment l'ajout de capacité de double couche $C_{\text {dif }}$ ou d'un élément à phase constant $(\mathrm{CPE})$ en parallèle avec l'impédance faradique est une simplification considérable. La variation en fréquence de la capacité de double couche induite par le signal alternatif de 
mesure d'impédance peut apporter des informations supplémentaires très utiles.

Enfin, nous avons illustré l'emploi de la méthode d'impédance à la préservation du patrimoine culturel en bronze recouvert d'une patine. L'amino-triazole peut être utilisé au lieu du benzotriazle reconnu toxique et cancérigène.

\section{Remerciements}

Cette contribution est dédiée à notre regretté "patron", M. Israël Epelboin, l'initiateur de cette nouvelle voie de recherche. Que les permanents, les post-doctorants, les doctorants et les stagiaires, jeunes et anciens ainsi que nos partenaires étrangers qui ont participé à cette grande aventure trouvent ici l'expression de notre profonde gratitude.

\section{Références}

[1] W. Schwenk, H.E. Buhler, Corros. Sci. 3 (1963) 261

[2] M. Prazak, K. Barton, Corros. Sci. 7 (1963) 159

[3] J.E.B. Randles, Discusions Faraday Soc. 1 (1947) 11

[4] C. Wagner, W. Traud, Z. Elektrochem. 44 (1938) 391

[5] K. Bonhoeffer, W. Jena, Z. Elektrochem. 59 (1951) 151

[6] M. Stern, A.L. Geary, J. Electrochem. Soc. 104 (1957) 56
[7] J. Tafel, Z. Physik. Chem. 50 (1905) 641

[8] J. Newman, J. Electrochem. Soc. 113 (1966) 501

[9] S. Chechirlian, P. Eichner, M. Keddam, H. Takenouti, H. Mazille, Electrochim. Acta 35 (1990) 1125

[10] I. Epelboin, M. Keddam, H. Takenouti, J. Appl. Electrochem. 2 (1972) 71

[11] R. Antaño-Lopez, M. Keddam, H. Takenouti, Electrochim. Acta 46 (2001) 3611

[12] I. Epelboin, P. Morel, H. Takenouti, J. Electrochem. Soc. 118 (1971) 1282

[13] M. Serghini-Idrissi, M.C. Bernard, F.Z. Harrif, S. Joiret, K. Rahmouni, A. Srhiri, H. Takenouti, V. Vivier, M. Ziani, Electrochim. Acta 50 (2005) 4699

[14] G L.J. Trettenhahn, G.E. Nauer, A. Neckel, Vibrationnal Spectrosc. 5 (1993) 85

[15] R.L. Frost, Spectrochim. Acta A 5 (2003) 1

[16] M. Bouchard-Abouchacra, Thèse, Paris 2001

[17] K. Rahmouni, N. Hajjaji, F.Z. Harrif, S. Joiret, H. Takenouti, Proceedings of 4th Croatian Symposium on Electrochemistry, 28 mai- $1^{\text {er }}$ juin, Primosten, HDKI, édité par L. Duic, Zagreb, 2006

[18] B. Trachli, Thèse co-tutelle, Université Pierre et Marie Curie (Paris) et Université Ibn Tofail (Kenitra, Maroc), Étude sur la corrosion du cuivre en milieu $\mathrm{NaCl} 0,5 \mathrm{M}$ et sa protection par des inhibiteurs organiques et des films polymères obtenues par électropolymérisation, soutenue le 27 octobre 2001, Kenitra Maroc

[19] L. Muresan, S. Varvara, E. StupnišekLisac, H. Otmacic, K. Marušic, S. HorvatKurbegovic, L. Robbiola, K. Rahmouni, H. Takenouti, Electrochim. Acta 52 (2007) 7770-7779 